\title{
Avaliação da capacidade funcional, força muscular e função pulmonar de pacientes amputados $e$ protetizados ao nível transfemural: estudo piloto
}

\author{
Evaluation of functional capacity, muscular strenght \\ and lung function of amputees and prothetizades \\ at transfemoral level: pilot study
}

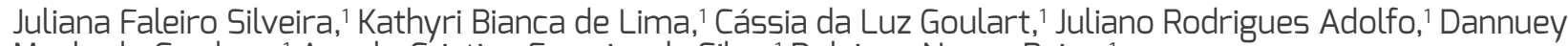
Machado Cardoso, ${ }^{1}$ Angela Cristina Ferreira da Silva, ${ }^{1}$ Dulciane Nunes Paiva.

Universidade de Santa Cruz do Sul (Unisc), Santa Cruz do Sul, RS, Brasil.

Recebido em: junho 2014 / Aceito em: março 2015

dulciane@unisc.br

\section{RESUMO}

A amputação é definida como a retirada total ou parcial de um membro, em que o coto é o responsável pelo controle da prótese durante o ortostatismo e a deambulação. A amputação pode causar déficit de equilíbrio, perda da funcionalidade e da força muscular periférica (FMP) do membro amputado bem como disfunção respiratória. Objetivos: Avaliar a capacidade funcional (CF), a FMP e a Força muscular respiratória (FMR) de indivíduos protetizados com amputação ao nível transfemural. Método: Avaliados indivíduos amputados em nível transfemural (GA) e indivíduos hígidos (grupo controle) (GC) com faixa etária de 18 a 60 anos. A CF foi avaliada pelo teste TimedUpandGo (TUG) e a FMP, através do teste de 01 Repetição Máxima (1RM) que avaliou os músculos extensores do joelho do membro não amputado. A FMR foi avaliada por manovacuometria digital. Resultados: Avaliados 13 indivíduos com média de idade de 40,69 anos e mediana de IMC 24 (22 - 35) Kg/m² (GA, $\mathrm{n}=6$; GC, $\mathrm{n}=7$ ). Os grupos se caracterizaram homogêneos quanto ao gênero, idade e IMC. A FMP e a FMR não diferiram entre os grupos tendo havido, entretanto, houve diferença significativa do TUG ( $p=0,001)$. Considerações finais: A capacidade funcional de indivíduos amputados transfemurais se encontra reduzida quando comparada com sujeitos hígidos. Não se encontrou redução significativa da FMP e FMR no grupo estudado.

Palavras-Chave: Amputação; Prótese; Força Muscular; Avaliação.

\section{ABSTRACT}

Amputation is defined as the total or partial withdrawal of a member where the stump is responsible for the prosthesis control during the standing position and walking. Amputation can cause balance disorders, loss of functioning and peripheral muscle strength (PMS) of the amputated limb as well as respiratory dysfunction. Objective: evaluate the functional capacity (FC), the PMS and respiratory muscle strength (RMS) of individuals fitted with transfemoral amputation level. Method: transfemoral amputated level subjects (experimental group - EG) and healthy individuals (control group - GC) aged 18-60 years were evaluated. FC was evaluated by the test Timed Up and Go (TUG) and PMS through 01 Maximum Repetition Test (1RM) that evaluated the knee extensor of not amputated limb. RMS was assessed by digital manometer. Results: We evaluated 13 subjects with a mean age of 40.69 years and median BMI of 24 (22-35) kg/m $/ \mathrm{m}^{2}$ (EG, $n=6 ; C G, n$ $=7$ ). The groups were characterized homogeneous in terms of gender, age and BMI. There was not difference in PMS and RMS between the groups, however, there was a significant difference in TUG ( $p=0.001)$. Final considerations: functional capacity of transfemoral amputees is low when compared to healthy subjects. There was no significant reduction in the PMS and RMS in this group.

Keywords: Amputation; Prosthesis; Muscle Strength; Evaluation. 


\section{INTRODUÇÃO}

O termo amputação se refere à retirada total ou parcial de um segmento corporal, geralmente cirúrgi$\mathrm{ca}^{1}$. O membro residual da amputação é denominado coto e ele é considerado como um novo membro, se tornando responsável pelo controle da prótese durante o ortostatismo e a deambulação. A amputação transfemural refere-se a toda amputação realizada entre a desarticulação do joelho e quadril, podendo ocorrer em três níveis: (i) nível transfemural em terço proximal, (ii) nível médio e (iii) nível distal. De acordo com o Instituto Brasileiro de Geografia e Estatística (IBGE), existem mais de 466.937 mil brasileiros sem um membro ou parte dele. Tais dados envolvem amputados e pessoas que já nasceram sem um membro ${ }^{2}$.

O amputado de membro inferior apresenta um gasto energético maior para a deambulação e este aumenta em relação ao nível da amputação ${ }^{3}$. Tais pacientes podem apresentar perda da capacidade funcional (CF) e da força muscular periférica (FMP) no membro amputado o que pode resultar em déficit de equilíbrio. O teste de Timed Up and Go (TUG) foi desenvolvido para quantificar a mobilidade física de idosos, podendo também avaliar o status funcional e equilíbrio de indivíduos amputados ${ }^{4}$.

Dentre as diversas formas de avaliação da força muscular periférica, o Teste de 01 Repetição Máxima (1RM) é um dos principais métodos para aferição da força muscular de um indivíduo, consistindo na determinação de carga máxima que um indivíduo é capaz de utilizar ao completar uma única repetição de determinado exercício ${ }^{5}$. Há escassez de relatos na literatura sobre se indivíduos amputados transfemurais apresentam disfunção respiratória devido à redução de sua locomoção. A avaliação da força muscular respiratória (FMR) nesses indivíduos permite possibilitar investigar as condições da força e o desempenho mecânico dos músculos da respiração ${ }^{6}$.

Assim, o objetivo do presente estudo foi o de avaliar o comportamento da capacidade funcional, da força muscular e da função pulmonar de indivíduos protetizados com amputação ao nível transfemural.

\section{MÉTODO}

Trata-se de um estudo piloto transversal do tipo casos-controle, realizado no Serviço de Reabilitação Física da Universidade de Santa Cruz do Sul Santa Cruz do Sul-RS, com aprovação do pelo Comitê de Ética e Pesquisa da instituição sob número de protocolo 10965612.7.0000.5343.

A amostra avaliada foi constituída por indivíduos sedentários, com idades entre 18 e 60 anos, de ambos os sexos, sendo alocados no Grupo Amputados (GA) composto por indivíduos amputados e protetizados ao nível transfemural, por no máximo 02 anos, que utilizassem próteses endoesqueléticas ou exoesqueléticas e que assinassem o TCLE. Os do Grupo Controle (GC) são indivíduos hígidos, que aceitaram o Termo de Consentimento Livre e Esclarecido (TCLE). Foram excluídos do estudo indivíduos com IMC $>40 \mathrm{Kg} / \mathrm{m}^{27,8}$, tabagistas, portadores de disfunções cardíacas, respiratórias ou do sistema locomotor.

\section{Timed Up and Go Test}

Para mensuração da CF, realizou-se o Teste Timed Up and Go (TUG) através de mini pedômetro digital (Mormaii ${ }^{\circledR}$, versão 2.0, China), que registrou o número de passos realizados pelo indivíduo analisado. Na posição sentada, o indivíduo foi posicionado a uma distância de $03 \mathrm{~m}$ de linha formada por fita adesiva fixada ao chão. $\mathrm{O}$ indivíduo permaneceu em posição sentada com tórax ereto, sendo orientados a levantar-se sem o apoio dos membros superiores (MMSS) percorrer 03 metros, retornando à cadeira e sentando-se sem o auxílio dos $\mathrm{MMSS}^{9}$.

\section{Teste de Uma Repetição Máxima (1RM)}

A avaliação da FMP foi realizada através do Teste de 1RM, tendo o mesmo sido empregue no membro não amputado. Foi utilizada cadeira extensora, onde foram acrescentados $5 \mathrm{~kg}$ a cada série de duas repetições, com intervalo de descanso de três minutos entre as séries, até que fosse encontrado o valor do $1 \mathrm{RM}$ do indivíduo ${ }^{10}$. Ressalta-se que a carga utilizada foi redimensionada a partir da tabela de Lombardi $^{11}$ até que o indivíduo realizasse uma repetição, tendo no máximo cinco tentativas para atingir a carga máxima. $O$ intervalo entre as tentativas foi de três minutos para que as reservas energéticas fossem restauradas ${ }^{12}$. Cada fase do movimento (fase concêntrica e fase excêntrica) teve uma duração de 2 segundos.

\section{Manovacuometria}

A medida da FMR foi realizada através de um manovacuômetro digital (MVD300, Globalmed ${ }^{\circledR}$, Campo $^{\circ}$ Grande,Brasil) com variação de $\pm 300 \mathrm{cmH}_{2} \mathrm{O}$, onde os voluntários permaneceram sentados, com o tronco em ângulo de $90^{\circ}$ em relação ao quadril, e os pés no chão e todas as aferições foram realizadas segundo as normas da American ThoracicSociety/EuropeanRespiratorySociety (ATS/ERS) ${ }^{13}$, sendo a pressão inspiratória máxima $\left(\mathrm{PI}_{\max }\right)$ obtida após expiração o volume residual (VR) seguida de um esforço inspiratório máximo. Para avaliação da pressão inspiratória máxima $\left(\mathrm{PE}_{\max }\right)$ os voluntários realizaram expiração até a capacidade pulmonar total (CPT), seguida de um esforço expiratório máximo. Os valores obtidos foram comparados com os previstos através das equações propostas para população brasileira ${ }^{13}$.

\section{Análise estatística}

A análise dos dados foi realizada através do programa Statistical Package for Social Sciences (SPSS 20.0, EUA). Para análise de normalidade da amostra foi realizado o teste Shapiro Wilk e para comparação dos dados antropométricos (exceto a variável gênero, que foi utilizado Teste de Qui-Quadrado), status funcional e função pulmonar entre os dois grupos foi realizado o Teste $U$ de Mann-Whitney. A associação entre o status funcional e a PImax foi avaliada pela correlação de Spearman. Para fins de significância estatística, foi considerado $\alpha 5 \%$, sendo os resultados expressos em $\mathrm{p}$ valor obtido.

\section{RESULTADOS}

A amostra foi composta por 13 indivíduos (GA: 
Tabela 1 - Características antropométricas, força muscular respiratória e 1RM dos grupos estudados.

\begin{tabular}{|c|c|c|c|}
\hline & Grupo Controle & Grupo Amputados & p-valor \\
\hline Sexo masculino, n (\%) & $3(43)$ & $4(67)$ & 0,688 \\
\hline Idade (anos) & $36(31-49)$ & $43,5(34,2-49,7)$ & 0,731 \\
\hline IMC $\left(\mathrm{Kg} / \mathrm{m}^{2}\right)$ & $24(22-35)$ & $22,5(18,7-29,2)$ & 0,731 \\
\hline $\mathrm{PI}_{\max }(\mathrm{cmH} 2 \mathrm{O})$ & $102(77-121)$ & $82(53-89,5)$ & 0,181 \\
\hline $\mathrm{PE}_{\max }(\mathrm{cmH} 2 \mathrm{O})$ & $148(92-184)$ & $118,5(74,5-138)$ & 0,101 \\
\hline 1 RM (kg) & $30(25-35)$ & $27,5(18,7-40)$ & 0,836 \\
\hline
\end{tabular}

$I M C=$ Índice de massa corpórea; $P I=$ Pressão inspiratória máxima; $P E=$ Pressão expiratória máxima; 1 RM= uma repetição máxima. Nivel de significância: $p<0,05$.

$\mathrm{n}=6$; GC: $\mathrm{n}=7$ ) sendo as causas das amputações as de etiologia traumática $(n=4)$, vascular $(n=1)$ e tumoral $(n=1)$. Deste total, três indivíduos amputaram o membro inferior esquerdo e três, o direito. Os grupos analisados se apresentaram homogêneos quanto à mediana do gênero, idade e IMC dos indivíduos alocados no estudo. A força muscular periférica e a FMR não apresentaram diferença estatística entre os grupos. Ressalta-se que os valores da $\mathrm{PI}_{\max }$ e da $\mathrm{PE}_{\max }$ no GA se mostraram menores que dos indivíduos do GC (Tabela 1).

O TUG revelou redução da capacidade funcional dos indivíduos amputados, em que o GC apresentou mediana de 8 (6 - 10) segundos, o que configura normalidade no teste e o GA apresentou mediana de 17,5 (13 - 22) segundos, o que configura um teste com alteração (Figura 1).

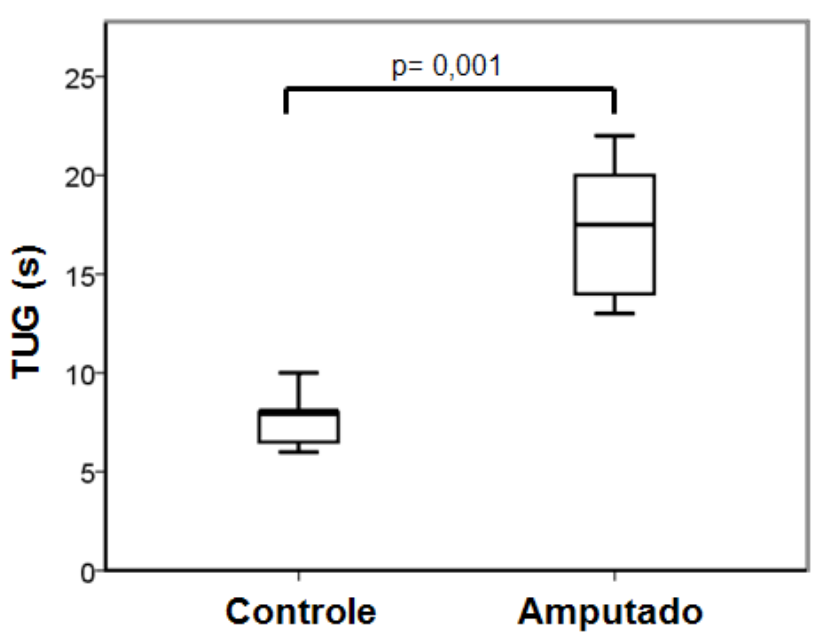

Figura 1 - Valores do Teste Timed Up and Go nos grupos avaliados. Nível de significância: $p<0,05$.

Encontrou-se correlação negativa quanto às variáveis TUG e FMR, ou seja, quanto menor o escore no teste de capacidade funcional (TUG), maior os valores de $\mathrm{PI}_{\text {max }}$.

\section{DISCUSSÃO E CONSIDERACִÕES FINAIS}

$\mathrm{Na}$ avaliação da capacidade funcional, encontrou-se diferença significativa entre os grupos estudados, em que se observa maior valor da mediana no $\mathrm{GC}$ em relação aos valores da mediana do GA. Tais resultados vêm ao encontro com outros estudos encontrados. Miller aplicou o mesmo instrumento em amputados transtibiais e transfemurais onde demonstra média de 14,5 segundos para o TUG ${ }^{14}$. Outro estudo vem de encontro aos nossos achados onde foi encontrado média de $10.4 \pm 3.7$ segundos em amputados transfemurais, porém este estudo alocou indivíduos com no mínimo cinco anos de uso da prótese e que pudessem deambular sem dificuldade, o que seria esperado devido ao maior tempo de adaptação proposto pelo autor do estudo ${ }^{15}$.

Ressalta-se que em amputações onde ocorre a retirada da articulação do joelho, a deambulação do indivíduo se torna mais difícil, o que pode comprometer severamente o status funcional do indivíduo. Nossos dados são corroborados por Aveiro et al. ${ }^{16}$, porém ressalta-se que o referido autor realizou o teste com indivíduos idosos, verificando que sujeitos com maior propensão à quedas realizaram o teste em maior tempo $(13,35 \pm 4,57$ segundos) e os menos propensos a quedas, em menor tempo $(11,71 \pm 3,61 \text { segundos })^{16}$

Quanto à força muscular periférica, não foi constatada diferença estatística entre os grupos, porém os indivíduos do GC apresentaram maior valor no teste de 1RM. Em um estudo realizado com três indivíduos amputados, utilizando eletromiografia e plataforma de força, foi descrita a atividade de força de reação ao solo de amputados transfemurais, sendo constatado aumento na atividade muscular na perna não amputada. Os amputados desenvolveram menor força de reação ao solo na perna amputada e longos períodos de atividade eletromiográfica na perna não amputada durante a marcha. Este estudo mostrou que indivíduos amputados desenvolvem mais força no membro contralateral à amputação ${ }^{17}$

Os valores de $\mathrm{PI}_{\max }$ e $\mathrm{PE}_{\max }$ mostraram-se reduzidos nos indivíduos amputados quando comparados ao dos indivíduos hígidos, porém sem relevância estatística da FMR entre os grupos. Supõe-se que isto ocorreu devido ao tempo de amputação dos indivíduos (de dois a três anos), bem como devido ao fato do nível de amputação apresentar-se distal ao gradil costal. Ressalta-se que devido à escassez de relatos na literatura especializada acerca desse tema, não foi possível estabelecer comparações com outros estudos quanto a esse aspecto.

As principais limitações do presente estudo foram o baixo tamanho amostral, onde resultados mais consistentes em relação ao teste de 1RM poderiam ser encontrados bem como a escassez de literatura sobre o referido tema. Ainda, o uso de diferentes tipos de próteses pelos pacientes avaliados podem ter interferido, nos desfechos avaliados.

Com isso, sugerimos que outros estudos sejam realizados nesta linha de investigação para que haja um maior aprofundamento e compreensão do assunto proposto. Dessa forma, a fisioterapia poderá desempenhar um papel mais ativo nas respostas às necessidades individuais de cada sujeito amputado.

O presente estudo permitiu concluir que a capaci- 
dade funcional de indivíduos amputados transfemurais se encontra prejudicado quando comparada com sujeitos hígidos, porém não se encontrou redução significativa da força muscular periférica e da força muscular respiratória nos grupos estudados, devido provavelmente às limitações do estudo.

Ao se discutir sobre a força muscular periférica e força muscular respiratória em amputados encontramos grande escassez de estudos, sugere-se que outros estudos sejam realizados nesta linha de investigação, para que se obtenha maior solidez quanto a estes achados na população em questão. Com um maior aprofundamento no tema referido, a fisioterapia poderá assim desempenhar ativamente seu papel frente às principais necessidades dos indivíduos amputados.

\section{REFERÊNCIAS}

1. Carvalho JA. Amputações de membros inferiores: em busca da plena reabilitação 2003.

2. Gomes ES, Coutinho RAM, Baraúna KMP, Valetine EF. Estudo Correlacional da Qualidade de Vida em Amputados de Membros Inferiores Transfemoral e Transtibial. Nova Fisio, Revista Digital, 2012; 15(87).

3. Gomes AIS, Ribeiro BG, Soares EA. Caracterização nutricional de jogadores de elite de futebol de amputados. Rev Bras Med Esporte, 2005; 11(1): 11-16.

4. Baraúna MA, Duarte F, Sanchez HM, Canto RST, MAlusá S, Campelo SCD. Avaliação do equilíbrio estático em indivíduos amputados de membros inferiores através da biofotogrametria computadorizada. Revista Brasileira de Fisioterapia, 2006; 10(1): 83-90.

5. Barros MAP, Sperandei S, Silveira JPCS, Oliveira CG. Reprodutibilidade no teste de uma repetição máxima no exercício de puxada pela frente para homens. Revista Brasileira de Medicina e Esporte, 2008; 14(4): 48-52.

6. Onaga $\mathrm{FI}$, Jamaml M, Ruas G, Lorenzo VAPD, Jamami LK. Influência de diferentes tipos de bocais e diâmetros de traqueias na manovacuometria. Fisioterapia em movimento,
2010; 23(2): 211-219.

7. Gottschall CBA, Shneider CD, Rabito El, Busnello FM. Guia prático de Clínica Nutricional: Tabelas, valores e referências. 2012.

8. Nacif M, Viebig RF. Avaliação antropométrica no ciclo da vida: uma visão prática. São Paulo: Editora Metha. 2011.

9. Ries JD, Echternach JL, Nof L, Blodgett MG. Test-Retest Reliability and Minimal Detectable Change Scores for the Timed "Up \& Go" Test, the Six-Minute Walk Test, and Gait Speed in People With Alzheimer Disease. Journal of the American Physical Therapy Association, 2009; 89(6): 69-79.

10. Pereira DAG, Faria BMA, Gonçalves RAM, Carvalho VBF, Prata KO, Saraiva PS. Relação entre força muscular e capacidade funcional em pacientes com doença arterial obstrutiva periférica: um estudo piloto. J Vasc Bras, 2011; 10(1): 26-30.

11. Lombardi VP, Beginning weight training : the safe and effective way. 1989.

12. Sakamoto A, Sinclair P.. Effect of movement velocity on the relationship between training load and the number of repetitions on bench press. Journal of Strength and Conditioning Research, 2006; 20(3): 523-527.

13. American Thoracic Society/European Respiratory S. ATS/ ERS Statement on respiratory muscle testing. Am J Respir Crit Care Med, 2002; 166(4): 518-624.

14. Miller WC, Deathe AB, Speechley M. Psychometric Properties of the Activities-Specific Balance Confidence Scale Among Individuals With a Lower-Limb Amputation. Arch Phys Med Rehabil, 2003; 84(5): 656-61.

15. Neder JA, Andreoni S, Lerario MC, Nery LE. Reference values for lung function tests. II. Maximal respiratory pressures and voluntary ventilation. Braz J Med Biol Res, 1999; 32(6): 719-27.

16. Aveiro MC, Driusso $\mathrm{P}$, Barham EJ, Pavarini $\mathrm{SCl}$, Oishi J. Mobilidade e risco de quedas de população idosa da comunidade de São Carlos. Ciência \& Saúde Coletiva.2012; 17(9): 2481-8.

17. Cerqueira ASO, Yamaguti EY, Mochizuki L, Amdio AC, Serrao JC. Ground reaction force and electromyographic activity of transfemoral amputee gait: a case series. Rev Bras Cineantropom Desempenho Hum, 2013; 15(1): 16-26. 